\title{
Probing Nanoparticle Geometry down to Sub-Nanometer Size: the Benefits of Vibrational Spectroscopy
}

Natalia Alyabyeva, Aimeric Ouvrard*, Abdoul-Mouize Zakaria and Bernard Bourguignon

Institut des Sciences Moléculaires d'Orsay (ISMO), CNRS, Université Paris-Saclay, F-91405 Orsay, France

ABSTRACT: Understanding the role of nanoparticle size and shape in the binding of molecules is relevant for a large range of applications like heterogeneous catalysis or molecular electronics. In this context, the geometry of Pd nanoparticles (NPs) has been studied over a wide range of size from very small clusters containing 4 atoms, up to large well faceted NPs made of more than 500 atoms. The NP height and

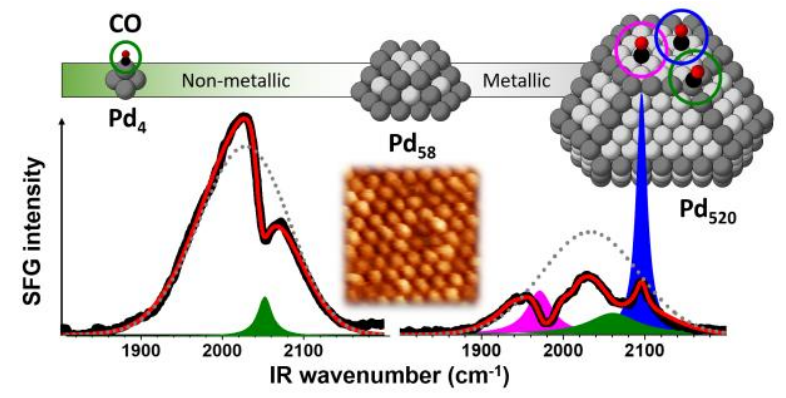
geometry are retrieved by combining scanning tunnelling microscopy (STM) and vibrational sum frequency generation spectroscopy (SFG) of adsorbed CO. SFG reveals to be highly sensitive to the geometry of NPs smaller than 100 atoms by identifying the nature of $\mathrm{CO}$ adsorption sites. This work highlights the fact that the growth of NPs can be followed layer by layer in the critical size range that corresponds to the transition from a non-metallic to a metallic state and to a marked minimum of CO adsorption energy reported in the literature. SFG shows that NP height remains of 2 Pd planes up to 30 atoms and that the two minima of adsorption energy correspond to the completion of the third and fourth layers.

KEYWORDS: SFG spectroscopy, STM microscopy, NP geometry, CO adsorption

INTRODUCTION: Ultrathin oxide supported metallic nanoparticles (NPS) have been widely investigated as model. ${ }^{1-4}$ The number of weakly coordinated atoms, their adsorption energy and reactivity change significantly with NP size..$^{5-7}$ It was observed that the exact number of atoms has a direct impact on reactivity that reveals the molecular behavior of very small clusters. Identifying the NP shape and counting active adsorption sites are then of great importance. Microscopy ${ }^{8,9}$ or X-ray diffraction ${ }^{10-12}$ provide valuable information on the NP shape with the support of density functional theory (DFT) calculations. ${ }^{13-14}$ Optical spectroscopy has also been used to probe adsorbed molecules in order to identify active adsorption sites and NP size effects. However, it does not allow deriving accurately the NP geometry and reactivity as a function of size because the size distribution is in general too broad on oxide (film or bulk) supported nanoparticles, where nucleation occurs on randomly distributed defect 
sites. In the past decade there appeared ultrathin films supporting NP arrays exhibiting a long-range order, high density and narrow-size distribution: $\mathrm{Al}_{2} \mathrm{O}_{3}{ }^{15-16}$ and $\mathrm{TiO}_{2}$ thin films ${ }^{17}$ and graphene single layer. ${ }^{11}$ Narrow size distribution, close to an ideal Poisson distribution, allows probing monometallic or core/shell NPs ${ }^{18}$ containing only a few atoms for catalysis ${ }^{6}$ and nanoelectronics. ${ }^{19}$ Sum frequency generation (SFG), as a surface sensitive vibrational spectroscopy, has been used to probe CO chemisorption with a high sensitivity. Pump-probe SFG allowed the study of energy transfer from Pd NP hot electrons to adsorbates, resulting in desorption and/or surface diffusion to specific sites ${ }^{20-23}$ in the case of a broad size distribution. In this work, NPs are grown along the "dot" hexagonal array of an ordered two-layer thick alumina layer on $\mathrm{Ni}_{3} \mathrm{Al}(111)$. We demonstrate the high sensitivity of SFG to detect $\mathrm{CO}$ on a wide range of NP sizes from a few atoms to several hundreds. Combined with its site selectivity, SFG CO spectroscopy allows probing the NP geometry with the support of scanning tunnelling microscopy (STM) and correlating NP geometry changes to reactivity.

EXPERIMENTAL METHODS: Sample growth and characterization were performed in a $2 \times 10^{-10} \mathrm{mbar}$ base pressure ultra-high vacuum chamber. Mechanically polished $\mathrm{Ni}_{3} \mathrm{Al}(111)$ single crystal (Surface Science Laboratory, Netherlands) was cleaned by repeated cycles of $15 \mathrm{~min} \mathrm{Ar}^{+}$sputtering ( $2 \mathrm{keV}, 15 \mu \mathrm{A} / \mathrm{cm}^{2}$ ) and annealing at $1123 \mathrm{~K}$ for $15 \mathrm{~min}$. $\mathrm{Al}_{2} \mathrm{O}_{3}$ ultrathin oxide film was grown using a recipe that was optimized to achieve long-range ordered NPs. ${ }^{24}$ The recipe consists in surface oxidation in $5 \times 10^{-8}$ to $2 \times 10^{-6} \mathrm{mbar}_{2}$ atmosphere ( $\sim 340 \mathrm{~L}$ dose) at $1143 \mathrm{~K}, \sim 140 \mathrm{~K}$ above the standard recipe reported in the literature, ${ }^{15,16,25,18}$ followed by surface annealing at $1193 \mathrm{~K}$. Surface temperature was monitored using a calibrated infrared pyrometer (IRcon, USA). NPs growth on $\mathrm{Al}_{2} \mathrm{O}_{3}$ was done at room temperature (RT) by Pd vapor deposition (99.98\% purity, Goodfellow, USA) at $0.18 \AA ̊ m i n$. At RT, Pd adatom diffusion length on $\mathrm{Al}_{2} \mathrm{O}_{3}$ remains larger than the distance between nucleation centers, guaranteeing a homogeneous nucleation of three-dimensional faceted NPs. ${ }^{26}$ Equivalent thicknesses ranging from 0.0025 to 2 monolayers (ML) of Pd were calibrated during the growth by a quartz microbalance and further calibrated by STM on specific terraces where layer by layer growth occurs. ${ }^{24}$ NP density and order were investigated using a STM operating at RT (ScientaOmicron, Germany). The amount of Pd atoms per NP was evaluated from the NP density and the evaporated Pd quantity. All investigated areas have been scanned twice in order to correctly identify NP diameter and height due to instable tip-surface interaction at the first pass. Origins of this effect, which are under investigation, are related to the oxygen dose used for $\mathrm{Al}_{2} \mathrm{O}_{3}$ growth and scanning parameters. SFG signals were produced on the 
sample by two $p$-polarized collinear laser beams, a near-infrared pulse $\left(806 \mathrm{~nm}, 7 \mathrm{~cm}^{-1} \mathrm{FWHM}, 3\right.$ ps duration) and an infrared pulse ( $2000 \mathrm{~cm}^{-1}, 150 \mathrm{~cm}^{-1} \mathrm{FWHM}, 140 \mathrm{fs}$ duration), covering the spectral region of CO internal vibration on Pd. SFG experiments were done at RT upon CO pressure varying from $10^{-9}$ to 1 mbar. Experimental details are given elsewhere. ${ }^{27-28}$ Prior to SFG, the sample was annealed at $523 \mathrm{~K}$ to desorb $\mathrm{CO}^{20}$ without impacting on NP ordering according to STM and literature. ${ }^{29}$

RESULTS AND DISCUSSION: STM images of Pd NPs grown on $\mathrm{Al}_{2} \mathrm{O}_{3} / \mathrm{Ni}_{3} \mathrm{Al}(111)$ corresponding to $\mathrm{Pd}$ deposits of 0.01, 0.05, 0.5 and $2 \mathrm{ML}(\sim 10, \sim 20, \sim 140$ and $\sim 520$ atoms of Pd per NPs) are presented in Figure 1a. For all deposits, NP nucleation occurs along the long-range ordered hexagonal "dot" superstructure of $4.12 \mathrm{~nm}$ periodicity, as confirmed by Fourier analysis, in agreement with literature. ${ }^{15,28,18}$ Statistical analysis of NP height distribution in $60 \times 60 \mathrm{~nm}^{2}$ regions are presented in Figure $1 \mathrm{~b}$ and gathered together with NP diameter in Table 1. The experimental most frequent NP heights measured by STM, are very close to the calculated ones assuming a half-sphere geometry, except for $0.01 \mathrm{ML}$ of Pd for which 1-2 atomic planes have been found by STM, while the calculated height is 2 layers. NP density from 0.0025 to $9 \mathrm{ML}$ of Pd is displayed in Figure 2a. The smallest amounts of $\mathrm{Pd}(\leq 0.1 \mathrm{ML}, \leq 30$ atoms/NP) correspond to the NP nucleation regime, where a fraction of nucleation sites are still empty. The growth regime above $0.1 \mathrm{ML}$ and below $2 \mathrm{ML}$ is characterized by an improved long-range periodicity as observed in self-correlation images of Figure 1a. The NP density raises up to $90 \%$ of nucleation centers of the "dot" structure $\left(6.54 \times 10^{12} \mathrm{~cm}^{-2}\right)$. These results are in agreement with the literature. ${ }^{18,29}$

Table 1. NP density $\mathrm{d}_{\mathrm{NP}}$ and geometrical parameters deduced by STM.

\begin{tabular}{lcccc}
\hline \hline $\mathrm{Pd}(\mathrm{ML})$ & 0.01 & 0.05 & 0.5 & 2 \\
\hline $\mathrm{d}_{\mathrm{NP}}\left(\times 10^{12} \mathrm{~cm}^{-2}\right)$ & $1.4 \pm 0.4$ & $4.6 \pm 0.3$ & $5.7 \pm 0.2$ & $5.6 \pm 0.2$ \\
\hline Diameter $(\mathrm{nm})$ & $1.1 \pm 0.2$ & $1.4 \pm 0.3$ & $2.4 \pm 0.3$ & $3.6 \pm 0.5$ \\
\hline Atomic planes & $1-2$ & $1-3$ & $3-5$ & $6-9$ \\
\hline Number of atoms & 10 & 20 & 140 & 520 \\
\hline \hline
\end{tabular}




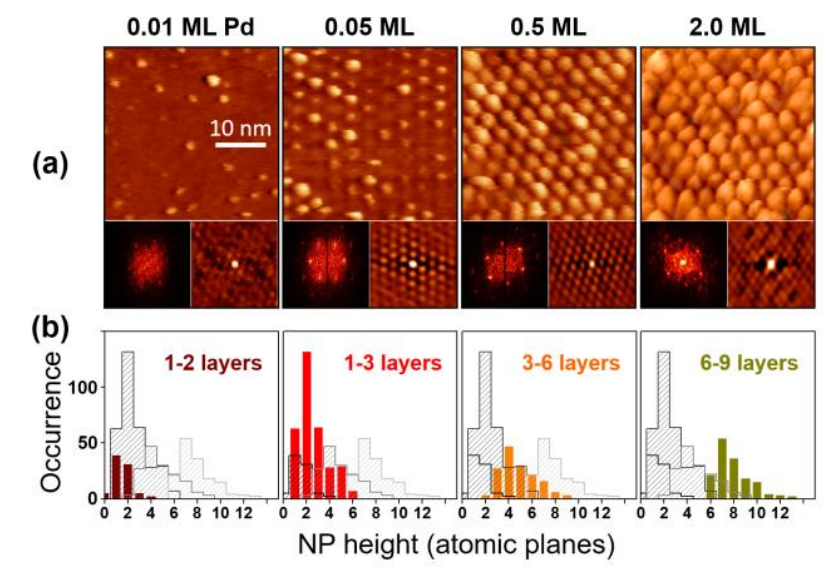

Figure 1. (a) STM images $\left(35 \times 35 \mathrm{~nm}^{2}, 1 \mathrm{~V}, 12 \mathrm{pA}\right)$ of Pd NPs grown on $\mathrm{Al}_{2} \mathrm{O}_{3}$ film on $\mathrm{Ni}_{3} \mathrm{Al}(111)$ for $0.01,0.05,0.5$ and 2 $\mathrm{ML}$ equivalent thicknesses of Pd. Fourier and selfcorrelation analysis are given under each image. (b) NP height distribution for the same Pd deposits as in (a).

Figure $2 \mathrm{~b}$ shows the experimental diameter, which is slightly larger than calculated model NPs of symmetric shapes having the appropriate number of atoms (Table A and B in Suppl. Information), corresponding to a half-sphere $(\mathrm{H}-\mathrm{S})$ or containing different numbers " $\mathrm{i}$ " of atomic layers $(\mathrm{i}-\mathrm{L})$. However, tip-surface convolution prevents measuring diameters smaller than $\sim 0.8 \mathrm{~nm}$, while the high density of particles hinders access to the oxide surface for the largest one. It indicates that the precision of STM measurements of NP width is not sufficient to determine accurately NP geometry or the actual number of layers for sizes below $\sim 100$ atoms.
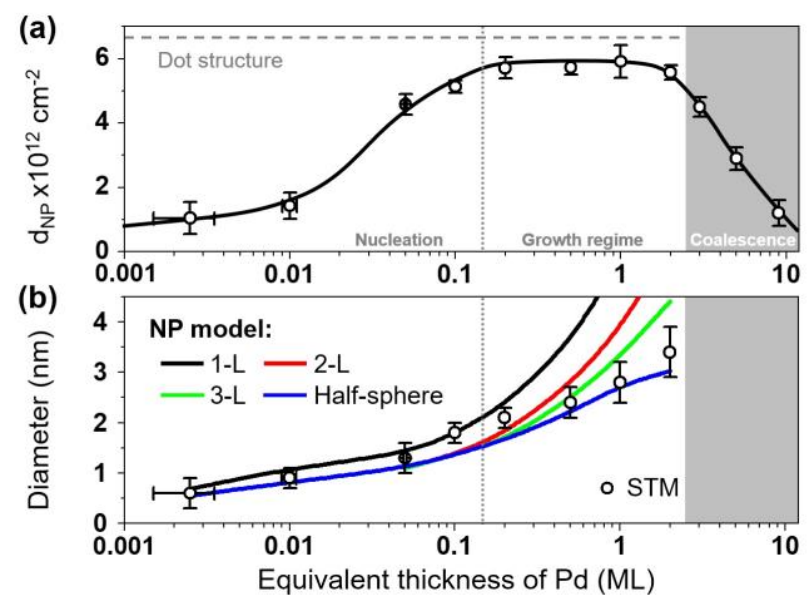

Figure 2. (a) NP density $d_{N P}$ as a function of Pd equivalent thickness (ML). Separation between nucleation and growth regimes (grey dotted lines) and dot structure density are 
highlighted (dashed line). (b) NP diameter ( $\mathrm{nm}$ ) as observed by STM (data points) and deduced from the different NP geometric models (color lines).

Figure 3 presents SFG spectra of CO adsorbed on Pd NPs under CO pressures of $10^{-8}$ and 1 mbar for $\mathrm{Pd}$ deposits of 0.0025 ( $\sim \mathrm{Pd}$ atoms/NP), $0.05,0.5$ and $2 \mathrm{ML}$. The result of spectrum fitting is shown, including a non-resonant signal arising from electronic states of NPs and the substrate in addition to $\mathrm{CO}$ vibrational bands. The fitting procedure is described in ref (28). The large range of pressure allows exploring different CO coverages: at $10^{-8} \mathrm{mbar}$, only strongly bonded $\mathrm{CO}$ are observed with an estimated CO coverage of $<0.4 \mathrm{ML}$, while at 1 mbar more sites are occupied, in the 0.6-0.7 ML range. Five vibrational bands have been observed (Table 2). Their intensities and frequencies depend strongly on NP size and CO pressure. For deposits smaller than 0.05 ML ( $<20$ atoms), only the band at 2035$2060 \mathrm{~cm}^{-1}$ has been observed (green band in Figure 3). This band is ascribed to edge sites, in agreement with previous work on $\mathrm{MgO}$ supported Pd NPs. ${ }^{20}$ According to DFT calculations for $\mathrm{Pd}_{148},{ }^{30}$ it can be attributed to on-top CO at edges $\left(2060-2070 \mathrm{~cm}^{-1}\right)$. The band increases significantly at $1 \mathrm{mbar}$, showing that all sites were not filled at $10^{-8}$ mbar. Other bands characteristic of facets appear at $0.05 \mathrm{ML}$ and above. They become larger than the edge band at $0.5 \mathrm{ML}$ ( 140 atoms). Indeed, adsorption sites on facets (bridge on (111) and (100) facets and on-top on (111) facets) can be assigned from their wellknown frequencies on single crystals. ${ }^{20,22}$ At low CO pressure, two bands appear at $1900-1920 \mathrm{~cm}^{-1}$ (orange), and 1950-1970 $\mathrm{cm}^{-1}$ (magenta). They are attributed to bridge sites on (111) and (100) facets, respectively. When the CO pressure is raised to $1 \mathrm{mbar}$, the "orange" band is almost replaced by the "magenta" band and an additional one "blue" band at $2090 \mathrm{~cm}^{-1}$. This is typical of CO compression on (111) surfaces, where this bridge + linear site combination was observed at a CO coverage of 0.6 ML. Only the magenta bridge band was observed at high pressure because (111) and (100) bridge bands nearly overlap at high coverage. A maximal coverage on (100) facets of $\sim 0.6 \mathrm{ML}$ similar to (111) is plausible. Frequencies are red shifted by a few tens wavenumbers with respect to single crystals, which is due to reduced dipolar coupling on NPs. For example, the observed blue shift of the bridge band around $1960 \mathrm{~cm}^{-1}$, as the NP size increases, is due to enhanced dipolar coupling as facets grow (Figure $3 b-d$ at 1 mbar). Note that relative intensities and frequencies may also vary because atomic and electronic structures change with size, especially for small NPs. The fifth band at $2010 \mathrm{~cm}^{-1}$ (grey band) is also present in the absence of NPs and is therefore assigned to CO on defects of the alumina film. 


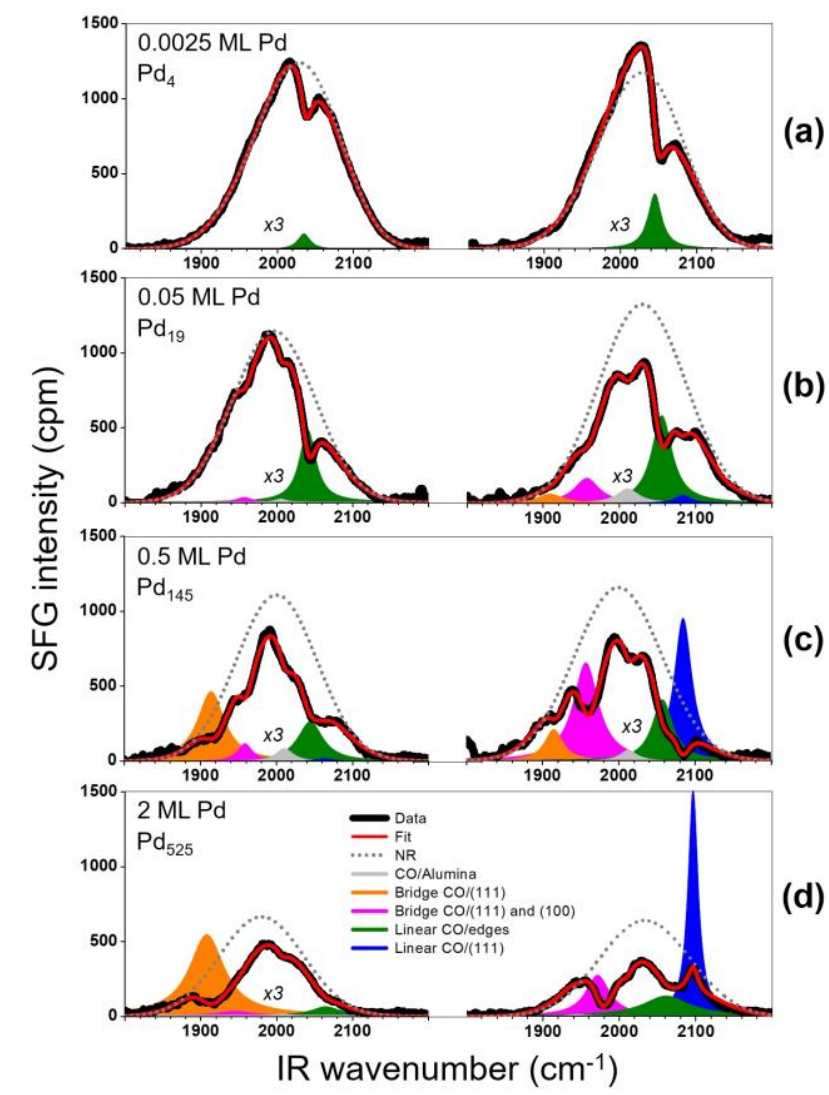

Figure 3. SFG spectra of CO adsorbed on Pd NPs for (a) 0.0025, (b) 0.05 , (c) 0.5 and (d) $2 \mathrm{ML}$ of Pd for two CO pressures ( $10^{-8}$ and 1 mbar). The different vibrational bands are referred to their color in the text. "NR" (grey dotted line) is the "non-resonant" signal. Its spectral shape is that of the IR laser. All band intensities have been multiplied by three for clarity except for (d) at 1 mbar.

Table 2. Assignment of $\mathrm{CO}$ vibrational band to adsorption sites.

\begin{tabular}{lccc}
\hline \hline Frequency $\left(\mathrm{cm}^{-1}\right)$ & Coordination & Location & Orientation \\
\hline $1900-1920$ & bridge & NP facet & $(111)$ \\
\hline $1950-1970$ & bridge & NP facet & $(100)$ or (111) \\
\hline 2010 & - & $\mathrm{Al}_{2} \mathrm{O}_{3}$ & $(111)$ \\
\hline $2035-2060$ & linear & $\mathrm{NP}$ edge & - \\
\hline 2090 & linear & NP facet & $(111)$ \\
\hline \hline
\end{tabular}

Using CO spectroscopy to identify adsorption sites as a probe of the NP geometry requires that NPS are not modified by $\mathrm{CO}$ adsorption. From molecular beam relaxation spectroscopy experiments, it has been concluded that Pd NPs remain stable at low CO coverage: (i) no particle sintering occurs upon CO 
exposure at low pressure and (ii) no change of adsorption energy has been observed after several adsorption/desorption cycles. ${ }^{6}$ In order to evaluate the impact of exposure in the mbar range, we have compared SFG spectra at low pressure before and after exposure at high CO pressure. Identical spectra have been obtained, suggesting that no irreversible geometry modification occurs, without excluding reversible changes. To track reversible changes, we have looked for spectroscopic changes that might occur at specific pressures and reveal a rearrangement of surface atoms. We can state that no significant NP geometry change occurs or that these possible reversible modifications take place faster than our acquisition time.

We have estimated the expected edge to edge + facet SFG band intensity ratio for symmetric atomic models of NP, ${ }^{31,13}$ assuming no geometry change upon CO adsorption. Details concerning NP geometry and atom and site counting are provided in Suppl. Information (Table A and B). SFG intensity ratio was fitted (Equation $A$ in Suppl. Information) by taking into account the number of sites and the $\mathrm{CO}$ coverage. We have assumed that the laser field direction at nanometer scale is constant, allowing to take into account the effect of CO dipole orientation on SFG intensity. ${ }^{32}$ The same hypothesis was done by the group of $\mathrm{H}$-J. Freund to estimate the correct $\mathrm{CO}$ site ratio of unsupported Pd particles. ${ }^{30} \mathrm{We}$ have first assumed that $\mathrm{CO}$ hyperpolarizability is site independent. Comparison of model and experimental relative SFG intensities at edges ( 1 mbar of CO) is shown in Figure 4 . No CO binding at facets for 0.0025 and $0.01 \mathrm{ML} \mathrm{Pd}$ (4 and 10 atoms) has been observed indicating that a CO molecule on a 4 atom NP prefers to bind on a linear site rather than on a bridge site. On a top facet of 3 atoms (case of a NP of 10 atoms with 2 planes) all atoms are at edges. Appearance of facet bands at $0.05 \mathrm{ML}$ $\mathrm{Pd}$ (19 atoms) is compatible with 7 atoms on the top facet in the 2-L case, excluding that a third layer (for which there would be no facet sites) has started to grow. At $0.1 \mathrm{ML}$ Pd (31 atoms), there are now 12 atoms on the top facet, corresponding to a further decrease of the fraction of edges. The third layer has not started. At $0.2 \mathrm{ML}$ of $\mathrm{Pd}$ (58 atoms) the third layer has started to grow, leading first to an increase of the edge fraction because the top facet contains 12 atoms, like at $0.1 \mathrm{ML}$, but with more atoms at edges. Above this size, the sensitivity of SFG relative intensity at edges to the number of atomic planes was lost, as shown by Figure 4. Quantitative adjustment of the hyperpolarizability at edges with respect to facets between 0.5 and $2 \mathrm{ML}$ Pd (between 140 and $520 \mathrm{Pd}$ atoms per NP), leads to a value of 0.75 , indicating that CO hyperpolarizability is little dependent on adsorption site for these NPs. The best NP models as deduced from the SFG edge site ratio are displayed in Figure 4 as a function of NP size. 


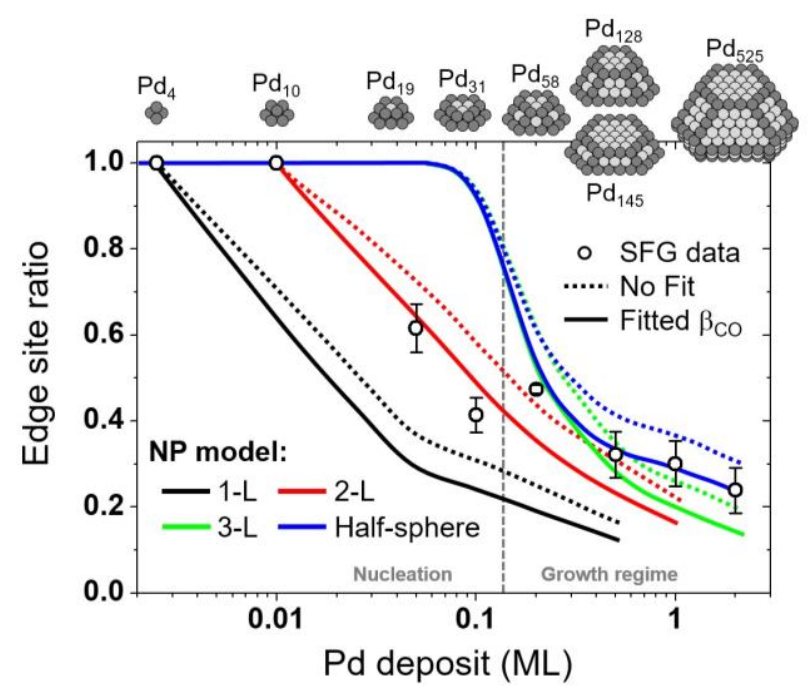

Figure 4. Edge site intensity ratio as observed by SFG (open circle) and calculated for the different NP geometry models, as a function of $\mathrm{Pd}$ deposited amount. The fitting parameter is CO hyperpolarizability $(\beta \mathrm{co})$ at edges. The best models are represented as a function of Pd deposit.

The height of NPs as a function of diameter, measured by STM and deduced from SFG, are compared in Figure 5a. They are in good agreement over the whole NP size range. STM provides an analog signal with error bars of the order of \pm 1 atomic plane, while SFG shows that the height is 2 planes until a diameter of $1.4 \mathrm{~nm}$ (30 atoms) and 3 planes until a diameter of $1.7 \mathrm{~nm}$ (60 atoms). The transition from the nucleation regime to the growth regime occurs between 1.4 and $1.7 \mathrm{~nm}$ (30 and 60 atoms). The transition of NP height from 2- to 3-layers corresponds to the beginning of the predominance of terrace sites over edge sites. Above $1.7 \mathrm{~nm}$ diameter (60 atoms), NPs have a facetted half-sphere shape. In Figure 5(b), the reported $\mathrm{CO}$ adsorption energy measured on $\mathrm{Pd} N \mathrm{NS}$ on $\mathrm{Al}_{2} \mathrm{O}_{3}$ is shown, ${ }^{6}$ exhibiting a marked decrease as a function of increasing size up to $\approx 1.7 \mathrm{~nm}$, with several local minima. This behavior was tentatively ascribed to the transition from non-metal to metal electronic structure. In the case of $\mathrm{TiO}_{2}$ supported Au NPs, the reduction of NP size below the metal to non-metal transition also leads to an increase of both the adsorption energy and the cluster reactivity. ${ }^{7}$ Pd NP size dependent adsorption energy reported in ref. (6) has been reproduced in Figure 5b and compared to our SFG results. First of all, the local adsorption energy minima match with transitions from 2 to 3,3 to 4 and 4 to 5 layers, meaning that CO binding strength is significantly modified by the number of atomic layers of the NP. Secondly, the frequency and bandwidth of the CO band at edges are plotted and their variation can be 
compared to adsorption energy changes with NP size. As for data in ref (6), the SFG data correspond to the smallest observable coverage. Indeed, upon $10^{-9}$ mbar of $\mathrm{CO}$, adsorption kinetics on Pd NPs can be followed from almost zero coverage to equilibrium coverage. ${ }^{20}$ Near zero coverage, the CO band frequency and bandwidth are not affected by dipolar coupling, so they reflect the pure coupling of $\mathrm{CO}$ with Pd NP. A fairly good anti-correlation between adsorption energy and frequency is found for 2 layer high NPs, while a correlation is observed for larger heights.
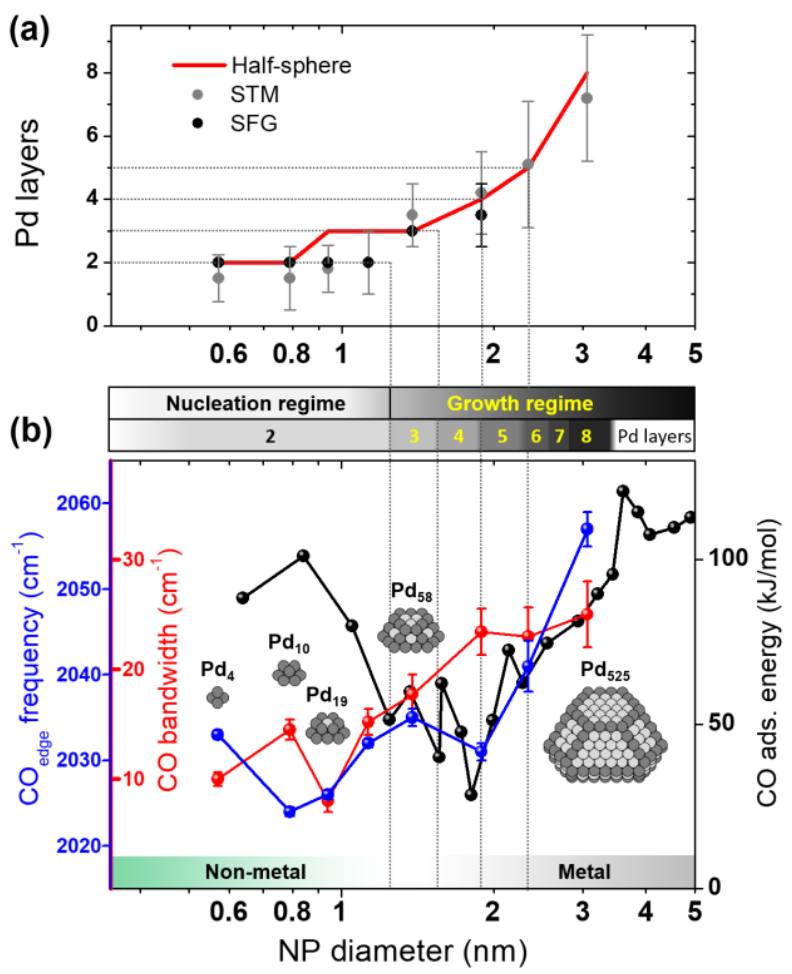

Figure 5. (a) NP height in Pd layers as a function of NP diameter measured by STM, deduced from SFG CO band intensity ratio and for the half-sphere model. (b) Deconvoluted frequency and bandwidth from SFG spectra of edge CO for Pd deposits from 0.0025 to $2 \mathrm{ML}$ compared to adsorption energy reproduced from ref. (6). CO bandwidth is defined as the half-width at half maximum of the lorentzian band profile used to fit SFG spectra. The number of Pd planes of the NP is shown above the figure.

In the refined Blyholder adsorption model of $\mathrm{CO}$ on metals, ${ }^{33}$ a lower frequency indicates a larger back-donation from the NP to the antibonding $2 \pi^{*}$ lowest unoccupied CO molecular orbital. During the growth of the second layer, the back-donation and the adsorption energy decrease together, while 
after the start of the third layer the back-donation decreases while the adsorption energy increases. The link between back-donation and adsorption energy relies strongly on the overlap of Pd orbitals that hybridize with $\mathrm{CO} 2 \pi^{*}$ orbital, hence on the coordination of adsorption sites (atop or bridge, facet or edge). The change from anti-correlation for 2-layer NPs to correlation for higher NPs thus indicates a change of the nature of edge sites between 2- and 3-layer high NPs. As height increases, important geometric parameters, like Pd-Pd distances, change due to relaxation of the strain imposed on the NP by the lattice mismatch with the oxide layer, as shown previously by DFT calculations for Pd NPs on MgO layer. ${ }^{20}$ The CO bandwidth continuously decreases for decreasing NP size with the exception of a marked local minimum for $\sim 1 \mathrm{~nm}$ diameter NPs ( 19 Pd/NP). Assuming that the bandwidth is homogeneously broadened, it measures the coupling strength between CO vibration and NP electronhole pairs. The coupling is expected to decrease at smaller NP size as the density of electronic states coupled to CO vibration becomes smaller. Thus the observed main tendency corresponds to expectation, while the exception for $\sim 1 \mathrm{~nm}$ NPs might correspond to an accidental reduced resonance between $\mathrm{CO}$ and NP electronic states. More detailed interpretation of these results would require DFT calculations.

In conclusion, we have used STM imaging and SFG vibrational spectroscopy to investigate Pd NP geometry and adsorption sites in the critical range of cluster size containing from $\sim 4$ to $\sim 520$ atoms, where the transition from molecular to metallic states takes place. Their combination allows following NP growth layer by layer. It reveals a transition from 2-layers to half-sphere geometry which corroborates previous measurements of adsorption energy. The completion of each layer is accompanied by measurable changes of the CO-NP coupling. SFG is particularly useful to probe NPS smaller than 100 atoms, where STM is reaching its limits. SFG observations are possible for two reasons: (i) the CO SFG intensity on small NPs is unexpectedly high, corresponding to a detection limit of $\sim 10^{-5}$ equivalent $\mathrm{ML}$ of $\mathrm{CO}$. We have observed previously a more moderate sensitivity enhancement of $10^{-4}$ for small Pd NPs on $\mathrm{MgO} / \mathrm{Ag}(100)$, attributed to local field enhancement; ${ }^{20,27}$ (ii) the accuracy and sensitivity are obtained thanks to the use of high density, narrow size distributed, long-range ordered NPs limiting inhomogeneous broadening. SFG was recently shown to be able to probe in realtime CO diffusion across edges and oxidation on larger NPs on $\mathrm{MgO} .^{23}$ It opens a way to follow reactions in-operando catalytic conditions using ultrafast vibrational spectroscopy for very well defined NP size and shape. 


\section{- ASSOCIATED CONTENT}

Additional information and figures in Supporting Information:

- Details concerning NP geometry and atom and site counting (Table A and B).

- $\quad$ Equation A used to fit SFG intensity ratio in Figure 4 of the manuscript.

\section{- AUTHOR INFORMATION}

*Corresponding author: aimeric.ouvrard@u-psud.fr

Notes: The authors declare no competing financial interest.

\section{- ACKNOWLEDGMENTS}

We thank the "Agence Nationale pour la Recherche" for funding this work (LEMON project ANR-15CE09-0007). We gratefully acknowledge Dr. Fabrice Charra (CEA-LEPO, Gif/Yvette, France) and Dr. Claude Viguier for active participation in the building of the STM used in this work.

\section{- REFERENCES}

(1) M. Haruta, "Size- and Support-Dependency in the Catalysis of Gold", Catal. Today, vol. 36, pp. 153-166, 1997.

(2) S. Bertarione, D. Scarano, A. Zecchina, V. Johanek, J. Hoffmann, S. Schauermann, M. M. Frank, J. Libuda, G. Rupprechter and H.-J. Freund, "Surface Reactivity of Pd Nanoparticles Supported on Polycrystalline Substrates As Compared to Thin Film Model Catalysts: Infrared Study of CO Adsorption", J. Phys. Chem. B, vol. 108, pp. 3603-3613, 2004.

(3) S. M. McClure and D. W. Goodman, "New Insights Into Catalytic CO Oxidation on Pt-Group Metals at Elevated Pressures", Chem. Phys. Lett., vol. 469, pp. 1-13, 2009.

(4) U. Heiz and U. Landman, "Nanocatalysis", Berlin Heidelberg: Springer-Verlag, 2007.

(5) H. Häkkinen, S. Abbet and A. Sanchez, "Structural, Electronic, and Impurity-Doping Effects in Nanoscale Chemistry: Supported Gold Nanoclusters", Angew. Chem. Int. Ed., vol. 42, no. 11, pp. 1297-1300, 2003.

(6) G. Sitja, S. Le Moal, M. Marsault, G. Hamm, F. Leroy and C. R. Henry, "Transition from Molecule to Solid State: Reactivity of Supported Metal Clusters", Nanoletters, vol. 13, pp. 1977-1982, 2013.

(7) D. C. Meier and D. W. Goodman, "The Influence of Metal Cluster Size on Adsorption Energies: CO Adsorbed on Au Clusters Supported on $\mathrm{TiO}_{2} "$, J. Am. Chem. Soc., vol. 126, pp. 1892-1899, 2004.

(8) O. H. Pakarinen, C. Barth, A. S. Foster and C. R. Henry, "Imaging the real shape of nanoclusters in scanning force microscopy", J. Appl. Phys., vol. 103, pp. 054313 1-7, 2008.

(9) A. Walton, J. Lauritsen, H. Topsøe and F. Besenbachera, "MoS2 nanoparticle morphologies in hydrodesulfurization catalysis studied by scanning tunneling microscopy", J. of Catal., vol. 308, pp. 306-318, 2013.

(10) G. Renaud, R. Lazzari, C. Revenant, A. Barbier, M. Noblet, O. Ullrich, F. Leroy, Y. Borensztein, J. Jupille, C. Henry, J. Deville, F. Scheurer, J. Mane-Mane and O. Fruchart, "In situ GISAXS towards a real time modelling of growing nanoparticles", Science, vol. 300, pp. 1416-1419, 2003. 
(11) D. Franz, S. Runte, C. Busse, S. Schumacher, T. Gerber, T. Michely, M. Mantilla, V. Kilic, J. Zegenhagen and A. Stierle, "Atomic Structure and Crystalline Order of Graphene-Supported Ir Nanoparticle Lattices", Phys. Rev. Let., vol. 110, pp. 065503 1-5, 2013.

(12) A. Bailly, G. Sitja, M.-C. Saint-Lager, S. Le Moal, F. Leroy, M. De santis, C. R. Henry and O. Robach, "Influence of palladium on the ordering, the final size and composition of Pd-Au nanoparticle arrays", J. Phys. Chem. C, vol. 121, pp. 25864-25874, 2017.

(13) C. Henry, "Morphology of Supported Nanoparticles", Prog. Surf. Sci, vol. 80, pp. 92-116, 2005.

(14) J. Goniakowski and C. Mottet, "Simulation of the growth of metal nanoclusters on the MgO(100) surface", Phys. Rev. B, vol. 81, pp. 155443 1-8, 2010.

(15) C. Becker, A. Rosenhahn, A. Wiltner, K. von Bergmann, J. Schneider, P. Pervan, M. Milun, M. Kralj and K. Wandelt, "Al${ }_{2} \mathrm{O}_{3}$-films on $\mathrm{Ni} 3 \mathrm{Al}(111)$ : a template for nanostructured cluster growth", New J. of Phys., vol. 4, pp. 75.1-75.15, 2002.

(16) M. Schmid, G. Kresse, A. Buchsbaum, E. Napetschnig, S. Gritschneder, M. Reichling and P. Varga, "Nanotemplate with Holes: Ultrathin Alumina on Ni3Al(111)", Phys. Rev. Lett., vol. 99, pp. 196104 1-4, 2007.

(17) L. Gavioli, E. Cavaliere, S. Agnoli, G. Barcaro, A. Fortunelli and G. Granozzi, "Template-assisted assembly of transition metal nanoparticles on oxide ultrathin films", Prog. Surf. Sci., vol. 86, pp. 59-81, 2011.

(18) M. Marsault, G. Hamm, A. Wörz, G. B. C. Sitja and C. Henry, "Preparation of regular arrays of bimetallic clusters with independent control of size and chemical composition", Faraday Discuss., vol. 138, pp. 407-420, 2008.

(19) S. Vlaic, L. Gragnaniello, S. Rusponi, A. Cavallin, F. Donati, Q. Dubout, C. Piamonteze, J. Dreiser, F. Nolting and H. Brune, "Interlayer exchange coupling in ordered Fe nanocluster arrays grown on $\mathrm{Al}_{2} \mathrm{O}_{3} / \mathrm{Ni}_{3} \mathrm{Al}(111)$ ", Phys. Rev. B, vol. 89, pp. 245402 1-9, 2014.

(20) A. Ouvrard, A. Ghalgaoui, C. Michel, C. Barth, J. Wang, S. Carrez, W. Zheng, B. Bourguignon and C. R. Henry, "CO Chemisorption on Ultrathin MgO-Supported Palladium Nanoparticles", J. Phys. Chem. C, vol. 121, no. 10, pp. 5551$5564,2017$.

(21) A. Ghalgaoui, A. Ouvrard, J. Wang, S. Carrez, W. Zheng and B. Bourguignon, "Electron to Adsorbate Energy Transfer in Nanoparticles: Adsorption Site, Size, and Support Matter", J. Phys. Chem. Letter, vol. 8, no. 12, pp. $2666-2671,2017$.

(22) G. Rupprechter, "Surface Vibrational Spectroscopy From Ultrahigh Vacuum to Atmospheric Pressure: Adsorption and Reactions on Single Crystals and Nanoparticle Model Catalysts Monitored by Sum Frequency Generation Spectroscopy", Phys. Chem. Chem. Phys., vol. 3, pp. 4621-4632, 2001.

(23) A. Ghalgaoui, R. Horchani, J. Wang, A. Ouvrard, S. Carrez and B. Bourguignon, "Identification of Active Sites in Oxidation Reaction from Real-Time Probing of Adsorbate Motion over Pd Nanoparticles", J. Phys. Chem. Lett., vol. 9, no. 18, pp. 5202-5206, 2018.

(24) N. Alyabyeva, A. Ouvrard, A. M. Zakaria, F. Charra and B. Bourguignon, "Transition from disordered to long-range ordered nanoparticles on $\mathrm{Al}_{2} \mathrm{O}_{3} / \mathrm{Ni}_{3} \mathrm{Al}(111) "$, Appl. Surf. Sci., vol. 444, pp. 423-429, 2018.

(25) A. Buchsbaum, M. De Santis, H. C. N. Tolentino, M. Schmid and P. Varga, "Highly ordered Pd, Fe, and Co clusters on alumina on $\mathrm{Ni} 3 \mathrm{Al}(111) "$ ", Phys. Rev. B, vol. 81, pp. 115420 1-12, 2010.

(26) G. Sitja, R. Omar Unac and C. R. Henry, "Kinetic Monte Carlo simulation of the growth of metal clusters on regular array of defects on insulator", Surf. Sci., vol. 604, pp. 404-408, 2010. 
(27) A. Ouvrard, J. Wang, A. Ghalgaoui, S. Nave, S. Carrez, W. Zheng, H. Dubost and B. Bourguignon, "CO Adsorption on Pd(100) Revisited by Sum Frequency Generation: Evidence for Two Adsorption Sites in the Compression Stage", J. Phys. Chem. C, vol. 118, pp. 19688-19700, 2014.

(28) J. Wang, H. Dubost, A. Ghalgaoui, W. Zheng, S. Carrez, A. Ouvrard and B. Bourguignon, "Effect of visible pulse shaping on the accuracy of relative intensity measurements in BBSFG vibrational spectroscopy", Surface Science, vol. 626, $\mathrm{p}$. 26-39, 2014.

(29) M. Marsault, G. Sitja and C. R. Henry, "Regular arrays of Pd and PdAu clusters on ultrathin alumina films for reactivity studies", Phys. Chem. Chem. Phys., vol. 16, p. 26458, 2014.

(30) I. Yudanov, R. Sahnoun, K. Neyman, N. Rösch, J. Hoffmann, S. Schauermann, V. Johanek, H. Unterhalt, G. Rupprechter, J. Libuda and H.-J. Freund, "CO Adsorption on Pd Nanoparticles: Density Functional and Vibrational Spectroscopy Studies", J. Phys. Chem. B, vol. 107, pp. 255-264, 2003.

(31) J. Goniakowski and C. Mottet, "Palladium nano-clusters on the $\mathrm{MgO}\left(\begin{array}{ll}1 & 0\end{array}\right)$ surface: substrate induced characteristics of morphology and atomic structure", J. Cryst. Growth, vol. 275, pp. 29-38, 2005.

(32) R. W. Boyd, Nonlinear Optics, Academic Press; Harcourt Brace Jovanovich, 1992.

(33) B. Hammer and J.K. Nørskov, "Theoretical surface science and catalysis-calculations and concepts", Adv. Catalysis, vol. 45, pp. 71-129, 2000. 